University of South Carolina

Scholar Commons

6-14-2011

\title{
Growth direction modulation and diameter-dependent mobility in InN nanowires
}

\author{
Goutam Koley \\ University of South Carolina - Columbia \\ Zhihua Cai \\ University of South Carolina - Columbia \\ Ehtesham Bin Quddus \\ University of South Carolina - Columbia \\ Jie Liu \\ University of South Carolina - Columbia \\ Muhammad Qazi \\ University of South Carolina - Columbia
}

See next page for additional authors

Follow this and additional works at: https://scholarcommons.sc.edu/phys_facpub

Part of the Physics Commons

\section{Publication Info}

Published in Nanotechnology, Volume 22, Issue 29, 2011, pages 1-8.

Koley, G., Cai, Z., Quddus, E.B., Liu, J., Qazi, M., \& Webb, R.A. (2011). Growth direction modulation and diameter-dependent mobility in InN nanowires. Nanotechnology, 22(29), 1-8. doi:10.1088/0957-4484/22/ $29 / 295701$

(c) 2011 IOP Publishing Ltd.

This Article is brought to you by the Physics and Astronomy, Department of at Scholar Commons. It has been accepted for inclusion in Faculty Publications by an authorized administrator of Scholar Commons. For more information, please contact digres@mailbox.sc.edu. 


\section{Author(s)}

Goutam Koley, Zhihua Cai, Ehtesham Bin Quddus, Jie Liu, Muhammad Qazi, and Richard A. Webb 


\title{
Growth direction modulation and diameter-dependent mobility in InN nanowires
}

\author{
Goutam Koley $^{1,3}$, Zhihua Cai ${ }^{1}$, Ehtesham Bin Quddus ${ }^{1}$, Jie Liu ${ }^{1}$, \\ Muhammad Qazi ${ }^{1}$ and Richard A Webb ${ }^{2}$ \\ ${ }^{1}$ Department of Electrical Engineering and Nanocenter, University of South Carolina, \\ Columbia, SC 29208, USA \\ 2 Department of Physics and Astronomy and Nanocenter, University of South Carolina, \\ Columbia, SC 29208, USA \\ E-mail: koley@engr.sc.edu
}

Received 25 January 2011, in final form 11 May 2011

Published 14 June 2011

Online at stacks.iop.org/Nano/22/295701

\begin{abstract}
Diameter-dependent electrical properties of InN nanowires (NWs) grown by chemical vapor deposition have been investigated. The NWs exhibited interesting properties of coplanar deflection at specific angles, either spontaneously, or when induced by other NWs or lithographically patterned barriers. InN NW-based back-gated field effect transistors (FETs) showed excellent gate control and drain current saturation behaviors. Both NW conductance and carrier mobility calculated from the FET characteristics were found to increase regularly with a decrease in NW diameter. The observed mobility and conductivity variations have been modeled by considering NW surface and core conduction paths.
\end{abstract}

(Some figures in this article are in colour only in the electronic version)

III-V nitride semiconductors (GaN, AlN, InN and their alloys) have attracted widespread research interest over the past two decades due to their unique material properties such as a wide range of direct bandgap, spontaneous and piezoelectric polarization, high electron mobility and chemical inertness [1-3]. InN exhibits many interesting properties of the III-nitride family, as well as some unique properties of its own, including a low bandgap $(\sim 0.7 \mathrm{eV})[4,5]$, downward surface band bending and surface electron accumulation [6-8]. In recent years, research interest has been directed towards studying $\mathrm{InN}$ nanowires (NWs) which exhibit many fascinating properties of $\mathrm{InN}$ thin films. Although several studies on InN NW synthesis [9], photoluminescence [10], electroluminescence [11], metal contacts [12], field effect transistors (FETs) [13] and $\mathrm{THz}$ emission [14] have been reported, many interesting properties of InN NWs have not yet been adequately understood. Slow progress in research on InN NWs can be attributed partly to synthesis issues such as low growth rate (only a few microns per hour) and poor material quality [15-18]. In particular, studies of transport

\footnotetext{
3 Author to whom any correspondence should be addressed.
}

properties, including measurements of electron mobility in $\mathrm{InN}$ NWs, have been difficult to perform due to poor material quality. When experimentally determined, the electron mobility for InN NWs have been found to be a few tens of $\mathrm{cm}^{2} \mathrm{~V}^{-1} \mathrm{~s}^{-1}[11,13]$, which is generally much smaller than that in large area thin films (usually $1000-2000 \mathrm{~cm}^{2} \mathrm{~V}^{-1} \mathrm{~s}^{-1}$ for carrier concentrations of $5 \times 10^{17}-10^{18} \mathrm{~cm}^{-3}$ ) [14, 19, 20]. This is in contradiction to GaN NWs which have electron mobilities comparable to or exceeding those of $\mathrm{GaN}$ thin films [21]. Such a low carrier mobility in InN NWs would limit their applications in sensors and high speed electronic devices. Synthesis of high-quality InN NWs with uniform cross section, long length and good crystalline property was first reported by Cai et al [22]. The carrier mobility in these NWs was generally found to be $\sim 50 \mathrm{~cm}^{2} \mathrm{~V}^{-1} \mathrm{~s}^{-1}$, which is somewhat higher than the mobility measured in other studies [13, 17], and further increased to $\sim 260 \mathrm{~cm}^{2} \mathrm{~V}^{-1} \mathrm{~s}^{-1}$ with application of a large negative gate bias.

An intriguing property of InN NWs observed in several past studies is the variation in conductance with $\mathrm{NW}$ diameter [23-26]. While some studies reported an increase in conductance with a decrease in NW diameter [23], and 
proposed theoretical models for the behavior [24], other studies found that the conductance can decrease with a decrease in NW diameter $[25,26]$. Clearly, the phenomenon of diameter-dependent transport in InN NWs needs to be examined more thoroughly, which can be highly significant for their applications in electronic, electromechanical and sensor devices. In this work, we have investigated structural and electrical properties of thin InN NWs with diameters of a few tens of nanometers. The NW-based FETs exhibited excellent gate control and drain current saturation. The carrier conductivity in the NWs was found to decrease with increasing NW diameter, due to variations in both carrier concentration and mobility, and was modeled considering two parallel conduction paths in the NWs. We also found that the NW direction can be altered systematically if other NWs or lithographic barriers are present in their growth path. Taken together, the novel structural and electrical properties of the NWs revealed through this study can spur widespread applications in nanoelectronic devices, nanoelectromechanical systems (NEMS), nanoscale chemical and biological sensors, and nanoprobes for scanning probe microscopes.

InN NWs were grown in a horizontal quartz tube furnace through direct reaction between metallic In and $\mathrm{NH}_{3}$ using an Au catalyst ( $\sim 20 \AA$ thick) deposited by thermal evaporation. The growth was performed by a VLS mechanism on an $\mathrm{n}^{+}$doped silicon (100) substrate with a $100 \mathrm{~nm}$ thick $\mathrm{SiO}_{2}$ layer. Several different catalysts including $\mathrm{Au}, \mathrm{Ni}, \mathrm{Cu}$ and $\mathrm{Co}$ were used for growth, but only Au was found to result in high-quality NW growth. Two separate quartz tubes were used to carry $\mathrm{NH}_{3}$ and $\mathrm{N}_{2}$ (carrying In vapor) to prevent In vapors from prereacting with $\mathrm{NH}_{3}$ and hindering $\mathrm{NW}$ growth. The growth was performed for $30 \mathrm{~min}$ at a temperature range of $650-700{ }^{\circ} \mathrm{C}$ and pressure of 50 Torr. $\mathrm{N}_{2}(99.998 \%$ purity) was passed over molten In (99.999\% purity) at the rate of 60-100 sccm, while the $\mathrm{NH}_{3}$ (99.9995\% purity) flow rate was maintained at 40$80 \mathrm{sccm}$. In our growth studies, we have observed that the best-quality NWs are obtained when they grow outwardly from lithographically patterned Au catalyst spots. If the Au catalyst film covered the entire substrate area, then three-dimensional (3D) growth is usually observed [22]. Figure 1(a) shows the scanning electron micrograph of NWs growing from a rectangular catalyst stripe pattern (only a section of one side is shown). The metal catalyst particles can be clearly seen at the apex of the NWs (see the magnified image in the image of figure 1(a)). Figure 1(b) shows a high resolution TEM image of a typical NW with a diameter of $\sim 20 \mathrm{~nm}$, with the hexagonal small area diffraction pattern shown in the inset. The EDX spectrum indicating the chemical composition is shown in figure 1(c). A small oxygen peak apart from the In and N peaks indicates the presence of an oxide shell layer. Such an oxide layer has also been observed earlier on InN NWs [22, 26].

Examining the various images of the NWs, we found that they exhibit two distinctive properties: (i) they are almost always coplanar, especially when growing on the plane of the substrate, and (ii) they change their growth direction either spontaneously or upon meeting an obstacle in their growth path [22]. Figure 2(a) shows an example of spontaneous bending of the NW, where it bends five times, each time at an angle of $\sim 30^{\circ}$. Barrier-induced multiple bending of $\mathrm{InN}$ NWs can be seen in figure 2(b), where the growth of the middle NW is guided by multiple deflections from two other guiding NWs with nanoscale precision. It should be noted here that, for multiple deflections to occur, it is necessary that all three NWs be in the same plane with precise positional accuracy better than a few nanometers, and the guiding NWs should be parallel to or at a slight angle to each other. The angles of deflection of the NWs were generally found to be close to $30^{\circ}$, $60^{\circ}$ or $90^{\circ}$. The geometrical precision of the NW growth and deflection indicates that this is not a random behavior exhibited by the NWs, but is determined by NW material properties. The bending of the NWs at multiples of $60^{\circ}$ can be understood from the fact that an InN wurtzite crystal has hexagonal symmetry with equivalent free energy of growth along equivalent $\langle 110\rangle$ or $\langle 1 \overline{1} 0\rangle$ directions (see the inset of figure 1(b) for the equivalent directions). The $30^{\circ}$ (or $90^{\circ}$ ) bending of the NWs happens due to a change in the NW growth direction from the $\langle 110\rangle$ to $\langle 1 \overline{1} 0\rangle$ direction (and vice versa), which is possible if the free energies in these growth directions are similar. To find out if the NWs can also be guided by lithographically patterned barriers, we deposited a $100 \mathrm{~nm}$ thick $\mathrm{SiO}_{2}$ barrier along with an adjacent $\mathrm{Au}$ catalyst spot as shown in figure 2(c). We find that an InN NW indeed deflects back and forth several times at specific angles $\left(\sim 90^{\circ}\right)$ forming nanoscale $\mathrm{V}$-shaped patterns. The NW exhibits three clear periods of deflection, and the process terminates as the NW deflection angle gets changed, most likely due to nanoscale roughness of the $\mathrm{SiO}_{2}$ barrier. If the catalyst pattern is deposited right at the edge of the lithographic barrier, and the growth is performed for a short enough duration, a single deflection resulting in a Vshaped structure can result, as shown in the inset of figure 2(c). These special V-shaped structures can be readily used to make $\mathrm{V}$-shaped nanocantilevers (VNCs) by etching the underlying $\mathrm{SiO}_{2}$ layer. The VNCs can be technologically very significant with potential applications in nanoelectromechanical systems (NEMS), scanning probes and sensor arrays. Such applications will, however, strongly depend on the electrical properties of the NWs, which need to be characterized in detail.

The electrical properties of the NWs were measured by fabricating FETs in a back-gated configuration, with the highly doped Si substrate acting as the back gate. The NWs used to fabricate FETs were grown from a central Au catalyst spot of $2 \mu \mathrm{m}$ diameter, which allowed only a few NWs to grow from it [22]. Following growth, pre-aligned ohmic contacts consisting of $\mathrm{Ti} / \mathrm{Al} / \mathrm{Ti} / \mathrm{Au}$ metal stacks were deposited at the center (covering the catalyst spot) as the source contact, and in a thin surrounding circular strip, as the drain contact (see figure 3(a)). Argon ion milling was performed before metal stack deposition to reduce ohmic contact resistance. This special device geometry helped the NWs to connect the source and the drain contacts with high probability, alleviating the need for meticulous post-growth alignment [27]. For these NWs the contact resistance was found to be quite small, usually $\sim 1 \mathrm{k} \Omega$ [28], which is much less than the NW resistance of hundreds of $\mathrm{k} \Omega$, so the device characteristics were obtained from two terminal (i.e. source-drain) measurements only. Our measured contact resistance agrees very well with that of 


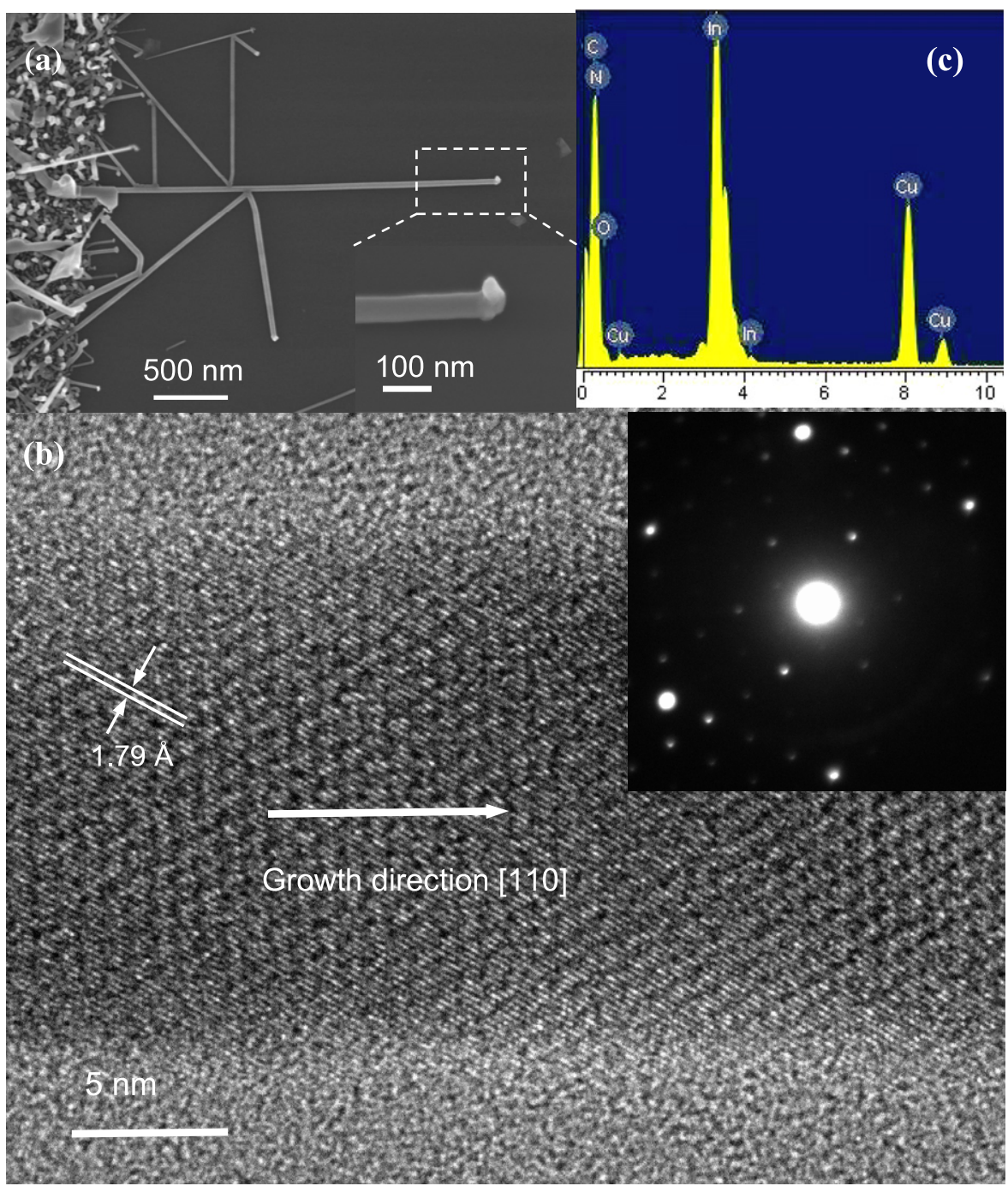

Figure 1. (a) SEM image of InN nanowires grown from a catalyst strip pattern, showing multiple deflections. Inset shows a magnified image of the NW with an Au catalyst particle at its apex. (b) High resolution TEM image of a single InN nanowire showing high crystalline structure. The growth is along the [110] direction. Inset shows the diffraction pattern along the zone axis [001]. (c) EDX spectrum corresponding to the nanowire shown in (b). The $X$-axis unit is keV, while the $Y$ axis is in arbitrary units.

$\sim 850 \Omega$ reported recently by Werner et al [26]. The SEM image of a typical fabricated device containing three sourcedrain connecting NWs is shown in figure 3(a). The diameters of the NWs were found to be 17,39 and $31 \mathrm{~nm}$ with a measurement uncertainty of $\pm 1 \mathrm{~nm}$ from SEM images. The $I_{\mathrm{d}}-V_{\mathrm{d}}$ and $I_{\mathrm{d}}-V_{\mathrm{g}}$ characteristics of the NW FET (measured in air at room temperature) are shown in figures $3(\mathrm{~b})$ and (c), respectively. The $I_{\mathrm{d}}-V_{\mathrm{d}}$ characteristics show excellent gate control, while the $I_{\mathrm{d}}-V_{\mathrm{g}}$ characteristics indicate a threshold voltage $V_{\text {th }}=-3.7 \mathrm{~V}$. The $I_{\mathrm{d}}-V_{\mathrm{d}}$ characteristics obtained for larger drain biases are shown in figure $3(\mathrm{~d})$. We find that drain current saturates beyond a certain $V_{\mathrm{d}}$, which, although very typical for FETs, has not so far been observed for any InN NW-based FET, possibly due to very high carrier density in these NWs $[11,13]$. However, the saturation of the drain current is important as it reveals the maximum current possible to obtain from a device under a given biasing condition. From figure 3(d), we observe that the saturation drain current reduces with larger $V_{\mathrm{d}}$, especially for lower gate biases. This is most likely due to heating of the NWs under high current, as observed earlier for GaN [27] and InAs [29] NWs.

The electron mobility $\mu_{\mathrm{e}}$ is related to the device transconductance $\mathrm{d} I_{\mathrm{d}} / \mathrm{d} V_{\mathrm{g}}$ by the equation [21]

$$
\mathrm{d} I_{\mathrm{d}} / \mathrm{d} V_{\mathrm{g}}=\mu_{\mathrm{e}}\left(C / L^{2}\right) V_{\mathrm{d}} .
$$

For an FET with multiple conducting NWs, equation (1) can be modified as (assuming a constant mobility for all the NWs)

$$
\mathrm{d} I_{\mathrm{d}} / \mathrm{d} V_{\mathrm{g}}=\mu_{\mathrm{e}}\left(\sum_{i=1}^{n} C_{i} / L_{i}^{2}\right) V_{\mathrm{d}} .
$$

Here $L_{i}$ and $C_{i}$ are the length and capacitance of the $i$ th NW in the FET and $V_{\mathrm{d}}$ is the drain bias. The capacitance $C_{i}$ is given as [30]

$$
C_{i}=\frac{2 \pi \varepsilon_{\mathrm{eff}} \varepsilon_{0} L_{i}}{\ln \left(2 h / r_{\mathrm{nw}, i}\right)},
$$

where $\varepsilon_{\text {eff }}$ is the effective dielectric constant accounting for the curvature of the NW, $h$ is the thickness of the oxide layer and 


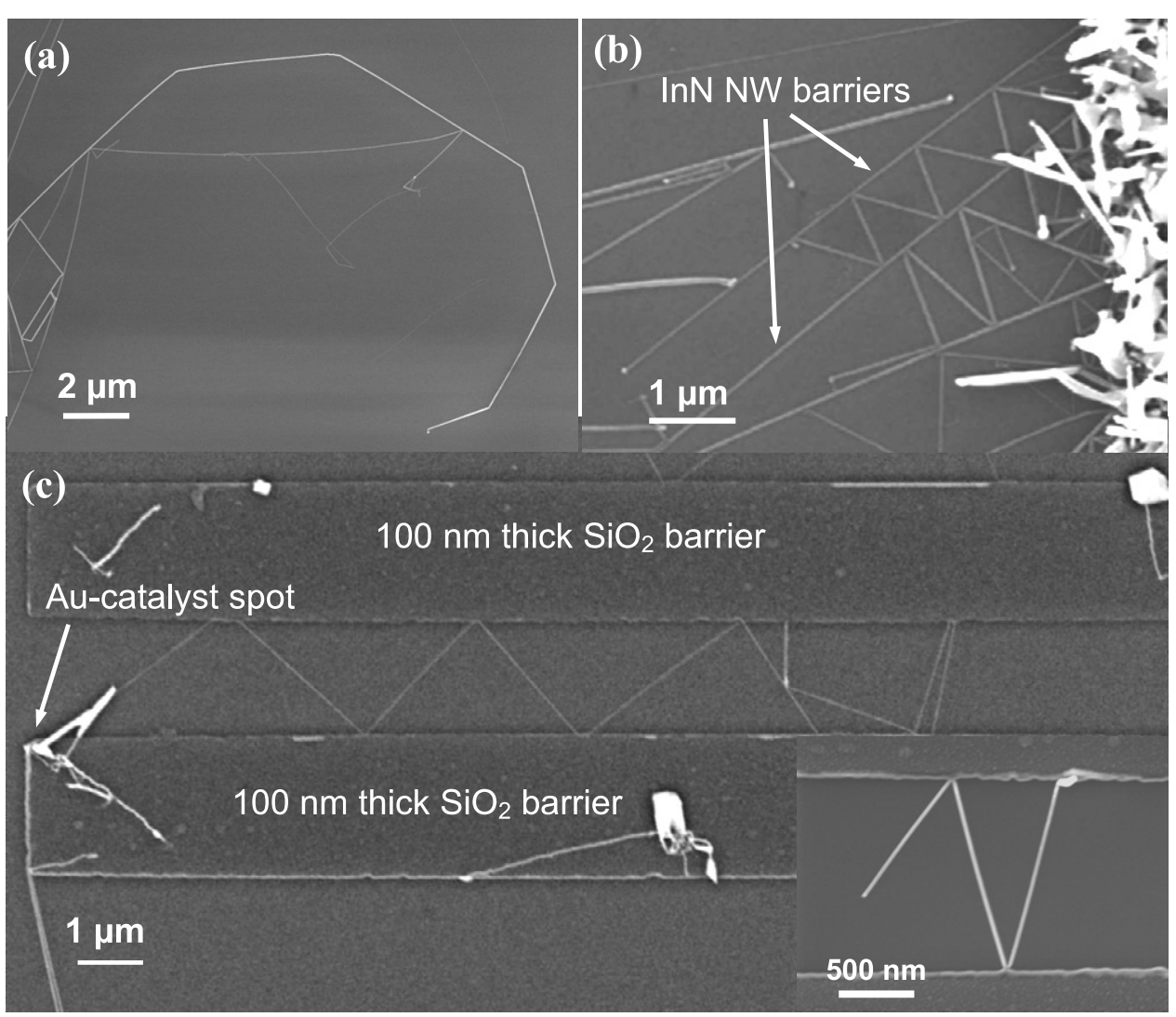

Figure 2. SEM images showing (a) multiple spontaneous bending of InN NW, as well as bending caused by (b) two InN NW guides, and (c) lithographically deposited $100 \mathrm{~nm} \mathrm{SiO} 2$ barriers. The inset of (c) shows a V-shaped NW structure formed by a single symmetric deflection from an $\mathrm{SiO}_{2}$ barrier.

$r_{\mathrm{nw}, i}$ is the radius of the $i$ th NW. Putting the value of the slope $0.33 \mu \mathrm{A} \mathrm{V}^{-1}$ obtained from the straight line fit to the middle section of the $I_{\mathrm{p}}-V_{\mathrm{bg}}$ curve (see figure 3(c)) into equation (2), the mobility and carrier concentration are calculated as $\sim 395 \mathrm{~cm}^{2} \mathrm{~V}^{-1} \mathrm{~s}^{-1}$ and $2.0 \times 10^{18} \mathrm{~cm}^{-3}$, respectively. It should be noted that the mobility and carrier concentration determined here are averaged over three NWs with different diameters, assuming the mobility is constant and independent of the NW diameter. However, while studying several FETs containing multiple NWs with different diameters we noticed that FETs having thicker NWs have lower average mobility than those having thinner NWs. The mobility of NW FETs containing only one NW, or multiple NWs with the same diameter, were also found to exhibit similar diameter-dependent mobility. This was an interesting observation, based on which we assumed a unique radius-dependent mobility $\mu_{\mathrm{e}}(r)$ for the NWs, which enabled us to extract mobility for many different diameters even from devices that contain multiple NWs (see the discussion below). Although dependence of NW conductance on diameter has been proposed earlier [23-25], to the best of our knowledge, dependence of mobility on NW diameter has not been proposed or investigated so far, mainly due to the difficulty in determining carrier mobility in InN NWs (through back-gate modulation of charges in an FET configuration) arising out of high carrier density $[11,13]$.

The overall transconductance for a set of $n$ NWs connecting the source and drain contacts follows from equation (1) as

$$
\frac{\partial I_{\mathrm{d}}}{\partial V_{\mathrm{g}}}=V_{\mathrm{d}}\left(\sum_{i=1}^{n} \frac{\mu_{\mathrm{e}}\left(r_{i}\right) C_{i}}{L_{i}^{2}}\right),
$$

where the capacitance $C_{i}$ for the $i$ th NW is given as $C_{i}=2 \pi \varepsilon_{\mathrm{eff}} \varepsilon_{0} L_{i} / \ln \left(2 h / r_{i}\right)$. Here $L_{i}, \quad r_{i}$ and $\mu_{\mathrm{e}}\left(r_{i}\right)$ are the length, radius and radius-dependent mobility of the $i$ th NW, respectively. Thus, separate equations for each FET can be obtained with the right-hand side containing a linear combination of $\mu_{\mathrm{e}}\left(r_{i}\right)$. Assuming the same electron mobility for NWs with similar diameters (varying within $\pm 2 \mathrm{~nm}$ ), these equations can be solved uniquely to obtain the mobility corresponding to each radius. Similarly, the drain conductance of the FET, which can be found out from the $I_{\mathrm{d}}-V_{\mathrm{d}}$ characteristics of the NWs, can provide information on the carrier concentration of individual NWs, if we also assume unique radius-dependent conductivity $\sigma\left(r_{i}\right)$. The total drain conductance of the FET is the sum of the individual conductance of the NWs in the FET, and is then given as

$$
\frac{\partial I_{\mathrm{d}}}{\partial V_{\mathrm{d}}}=\sum_{i=1}^{n} \frac{\sigma\left(r_{i}\right) A_{i}}{L_{i}}=\sum_{i=1}^{n} \frac{n_{i} q \mu_{\mathrm{e}}\left(r_{i}\right) A_{i}}{L_{i}}
$$

Here $\sigma\left(r_{i}\right)$ and $A_{i}$ are the radius-dependent conductivity and cross-sectional area of the $i$ th NW. Once again, assuming NWs with similar radii (varying within $\pm 1 \mathrm{~nm}$ ) have similar 

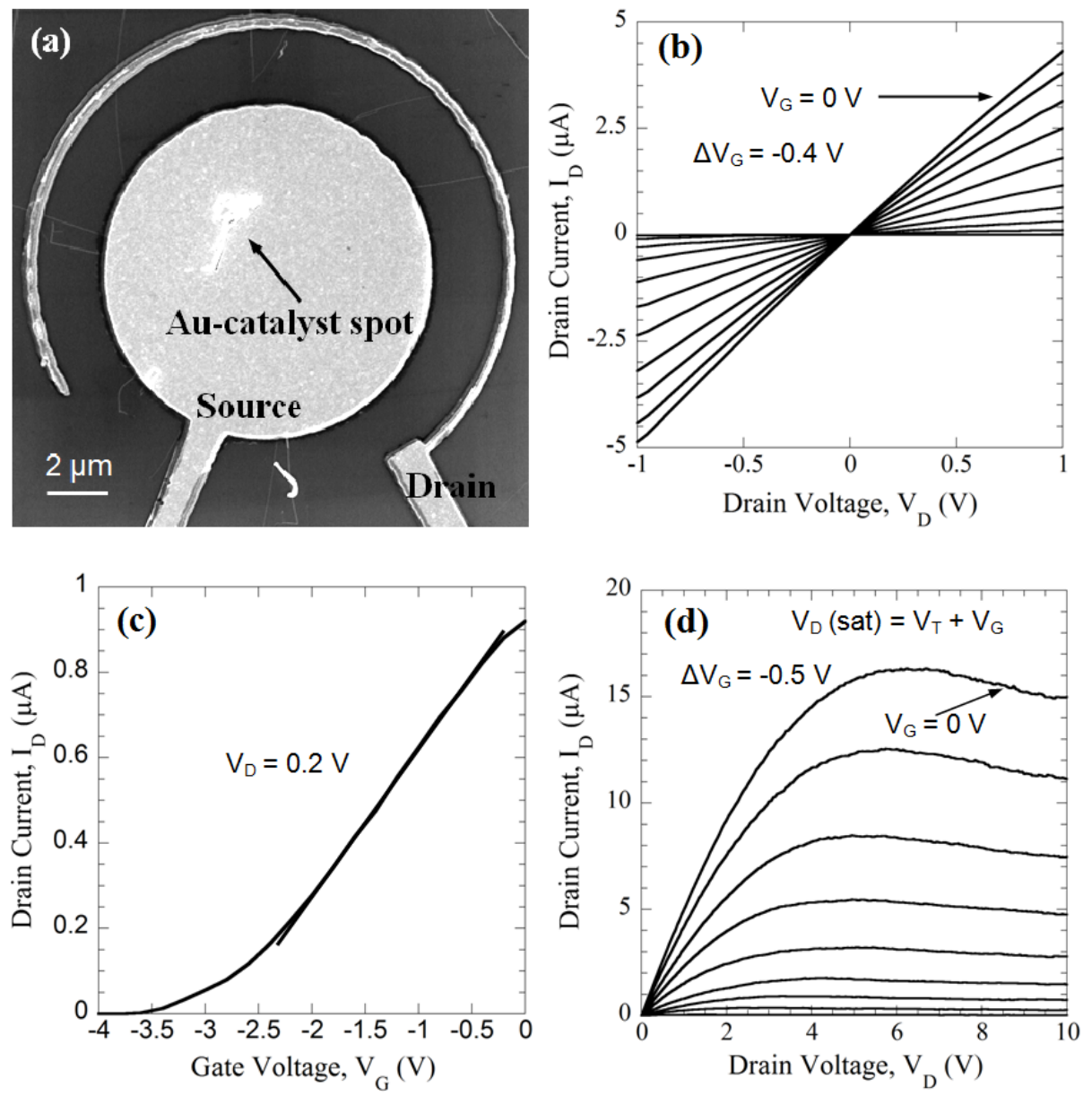

Figure 3. (a) SEM image of a three-NW FET in a back-gated configuration. (b) $I_{\mathrm{d}}-V_{\mathrm{d}}$ curves for the InN NW FET at gate bias voltages between 0 and $-3.6 \mathrm{~V}$ at intervals of $-0.4 \mathrm{~V}$. (c) An $I_{\mathrm{d}}-V_{\mathrm{g}}$ curve measured at $V_{\mathrm{d}}=0.2 \mathrm{~V}$, with the $\mathrm{d} I_{\mathrm{d}} / \mathrm{d} V_{\mathrm{g}}$ slope shown by a straight line. (d) $I_{\mathrm{d}}-V_{\mathrm{d}}$ saturation curves measured for gate bias voltages between 0 and $-4.0 \mathrm{~V}$ at intervals of $-0.5 \mathrm{~V}$.

conductivity, they can be calculated as a function of radius from the conductance data of all the devices (similar to the calculations of $\mu_{\mathrm{e}}\left(r_{i}\right)$ discussed above).

To verify the proposed idea of diameter-dependent mobility, NW FETs were fabricated in two separate batches several months apart. In the first batch, there were three FETs with either one NW or two NWs with the same diameter. There were five other FETs containing multiple NWs with different diameters. By assuming similar mobility for NWs with diameters within $\pm 2 \mathrm{~nm}$, the mobility values were solved from equation (4). The second batch of five devices, fabricated several months after the first batch, had either single NW or two NWs with the same diameter. The mobility $\mu(r)$ of the NWs plotted as a function of their diameter is shown in figure 4(a), with the mobility values obtained from FETs with single NW (or with two NWs with the same diameter) shown with separate symbols from the ones calculated from multiple-NW devices using equation (4). As we can see, the mobility decreases regularly as the NW diameter increases from 12 to $50 \mathrm{~nm}$. The reduction is very steep from 12 to $\sim 35 \mathrm{~nm}$, after which the change is slower. Interestingly, the variation of $\mu_{\mathrm{e}}(r)$ is very similar regardless of whether it is calculated from single NW or from multiple-NW FETs (using equation (4)), which clearly supports our proposition of diameter-dependent electron mobility. The conductivity $\sigma(r)$ plotted as a function of NW diameter is shown in figure 4(b). Once again we observe a trend of decreasing conductivity with increase in NW diameter. However, the variation is not as regular as that for mobility. The variation of carrier concentration $n(r)$ with $\mathrm{NW}$ diameter was determined using the relationship $\sigma\left(r_{i}\right)=\sigma\left(r_{i}\right) n\left(r_{i}\right) q$ for the $i$ th radius, and is shown as an inset in figure 4(b). Like $\sigma(r)$ and $\mu(r), n(r)$ also increases with decrease in NW diameter, but not as regularly as mobility. Since conductivity and carrier concentration varies as the square of the NW radius (as opposed to mobility where the NW radius appear in a logarithmic term), their variations due to inaccuracies in diameter can be quite large. This could be partly responsible for the less regular variation of the conductivity and carrier concentration with NW diameter compared to mobility.

It is worth mentioning here that, in most of the studies reported so far on electrical characterization of InN NWs, only the variation in conductivity with diameter has been reported, but not carrier mobility, since carrier mobility could not be 

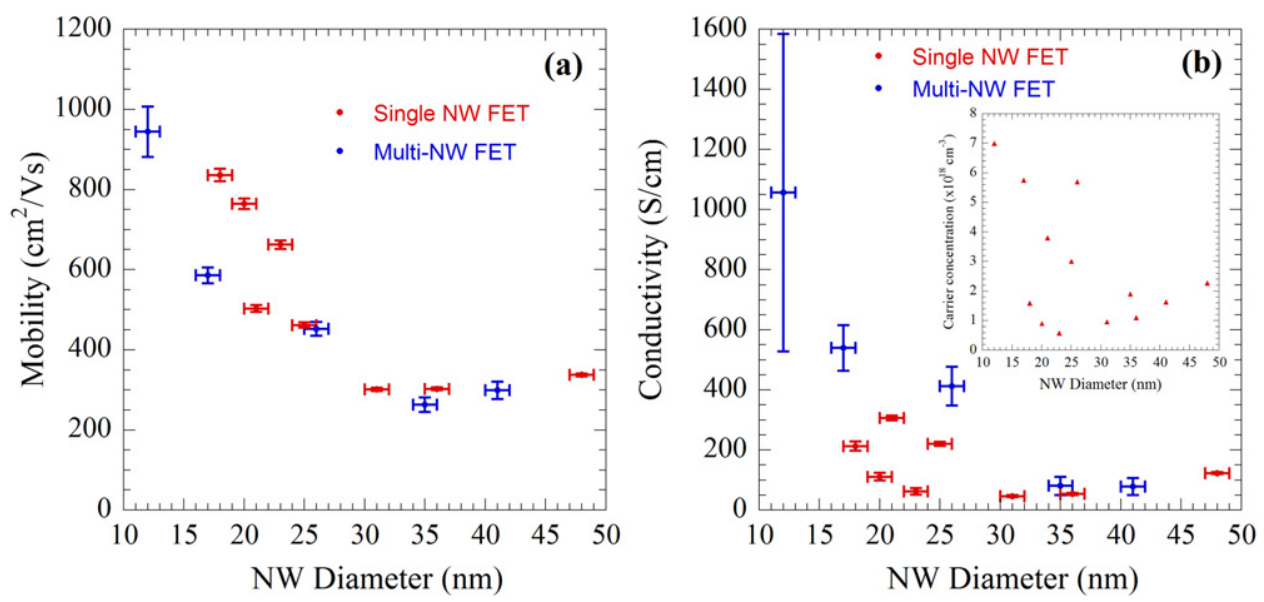

Figure 4. Variation of (a) mobility and (b) conductivity of InN NWs as a function of the diameter obtained from single and multi-NW FETs. The respective error bars are also shown. Inset of (b) shows the variation in carrier concentration with NW diameter.

determined experimentally in most cases. Therefore, it was difficult to conclude whether the carrier concentration, or the carrier mobility, or both, were responsible for the observed conductivity variation with NW diameter. Our experimental results reveal that both carrier concentration and mobility changes strongly with NW diameter and contributes to the conductivity variation. The measurement uncertainty for NW diameter and diameter grouping would lead to uncertainty in the calculated mobility, carrier concentration and conductivity values. For single-NW devices, the error is mostly from the uncertainty in diameter measurement from SEM images of $\pm 1 \mathrm{~nm}$. For multi-NW devices, the errors are larger and are contributed by diameter measurement uncertainty and the effect of grouping. The calculated error bars in mobility and conductivity are shown in figures $4(\mathrm{a})$ and (b).

The observed variation of carrier mobility with NW diameter was modeled by assuming two parallel conduction channels in InN NWs, one formed by the surface-accumulated electrons and the other by the core electrons, following earlier propositions $[24,26]$. Accumulation of electrons at the surface of InN thin films has been experimentally verified [6-8] and the same is also believed to hold true for InN NWs [11, 31, 32]. In our model, we consider that the surface electrons are confined to a depth of only a few nanometers, and have different densities and mobilities compared to the core electrons. Since the confinement of the carriers is determined by the Fermi pinning at a level above the conduction band [7, 8], when the diameter of the NW changes, the distribution profile of the carriers with distance beneath the surface can generally be expected to remain the same. For InN thin films, this charge distribution has been determined numerically by solving Poisson's equation within a modified Thomas-Fermi approximation [6]. For our simulations, we approximated the carrier profile reported in [5] to describe the variation of surface carrier density $n_{\mathrm{s}}$, which is found to approximately vary as $n_{\mathrm{s}}=n_{0} \mathrm{e}^{-\sqrt{x / 4}}$, where $x$ is the distance inside the surface of the NW in $n m$ and $n_{0}$ is the density (areal) of charges at the NW surface. The total surface carriers denoted as $N_{\mathrm{s}}$ and the total core carriers denoted as $N_{\mathrm{b}}$ are given as

$$
N_{\mathrm{s}}=L \int_{0}^{r_{0}} 2 \pi(R-x) n_{\mathrm{s}}(x) \mathrm{d} x, \quad N_{b}=n_{\mathrm{b}} \pi\left(R-r_{0}\right)^{2} L .
$$

Assuming that the surface and core electrons $N_{\mathrm{s}}$ and $N_{\mathrm{b}}$ move with a mobility of $\mu_{\mathrm{s}}$ and $\mu_{\mathrm{b}}$, respectively, the average mobility $\mu_{\text {eff }}$ is given as $\mu_{\mathrm{eff}}=\left(N_{\mathrm{s}} \mu_{\mathrm{s}}+N_{\mathrm{b}} \mu_{\mathrm{b}}\right) /\left(N_{\mathrm{s}}+N_{\mathrm{b}}\right)$. The conductivity is then calculated as $\sigma=q \mu_{\mathrm{eff}}\left(N_{\mathrm{s}}+\right.$ $\left.N_{\mathrm{b}}\right) /\left(\pi R^{2} L\right)$. The simulated electron mobility and conductivity as a function of NW diameter are shown in figure 5. In our simulations, we used $\mathrm{r}_{0}=2 \mathrm{~nm}, n_{\mathrm{b}}=5.67 \times 10^{18} \mathrm{~cm}^{-3}$, $\mathrm{n}_{0}=1.15 \times 10^{19} \mathrm{~cm}^{-3}, \mu_{\mathrm{s}}=1400$ and $\mu_{\mathrm{b}}=10 \mathrm{~cm}^{2} \mathrm{~V}^{-1} \mathrm{~s}^{-1}$, to obtain the least-squares fit to the mobility data. Interestingly, the mobility of the surface electrons of $1400 \mathrm{~cm}^{2} \mathrm{~V}^{-1} \mathrm{~s}^{-1}$ used in our simulations is much larger than the mobility expected for thin films for $n=5 \times 10^{18}-10^{19} \mathrm{~cm}^{-3}$, which should only be a few $100 \mathrm{~cm}^{2} \mathrm{~V}^{-1} \mathrm{~s}^{-1}$, based on measurements on $\mathrm{InN}$ thin films $[14,19,20]$. The high mobility could be due to better screening of charge carriers from remote ionized impurities by a high density of surface electrons, which is generally observed for two-dimensional electron gas (2DEG) systems [33, 34]. The core carrier mobility of $10 \mathrm{~cm}^{2} \mathrm{~V}^{-1} \mathrm{~s}^{-1}$ is comparable to what has been generally measured on larger diameter $\mathrm{InN}$ NWs $[11,13]$.

From figure 5(a), we observe that the carrier mobility calculated from experimental data fits quite well with the mobility versus diameter plot determined from equation (6). However, measured NW conductivity deviates significantly from the simulated values as seen in figure 5(b). This could be partly due to the presence of a few-nanometer-thick indium oxide layer on the NW surface, which would underestimate the conductivity of the NWs by increasing the NW diameter used in our calculations compared to the actual diameter of the conducting core. Our TEM images have shown the presence of these oxide layers [22], which have been observed by other researchers as well [26]. For mobility calculations, the diameter appears in the logarithmic term in equation (3), so small deviations do not cause a significant error in the 

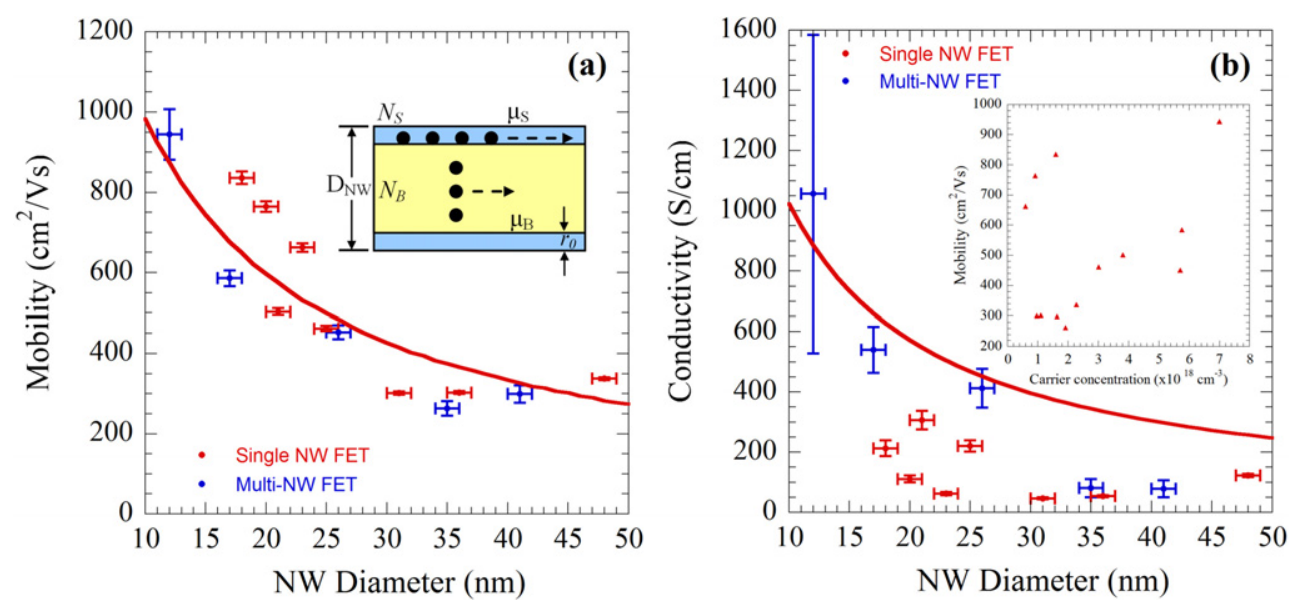

Figure 5. Variation of (a) mobility and (b) conductivity of InN NWs as a function of NW diameter fitted with diameter-dependent mobility and conductivity obtained from theoretical modeling (solid lines). Inset of (b) shows the variation of mobility with carrier concentration.

calculated mobility. The inset of figure 5(b) shows the variation of mobility with carrier concentration in the NWs. As we can see, the mobility increases fairly monotonically with the increase in carrier concentration, except for a few data points (i.e. 18, 20 and $23 \mathrm{~nm}$ diameters) that belong mostly to the NW FETs fabricated in the latter batch, where NWs might have developed a thicker overgrown oxide layer. In spite of some deviations, the overall trend clearly supports our model, which predicts higher mobility and carrier concentration for lower diameter NWs, where properties of the surface electrons dominate (which, according to our model, has higher density and mobility than the bulk electrons). It should be noted that the increase in mobility with carrier concentration is quite unique, and similar to that observed for 2DEG systems in modulation-doped heterostructures $[33,34]$. Since in these systems the mobility is mainly determined by remote ionized impurity scattering, it is possible that the latter also plays a dominant role in determining surface electron transport in the InN NWs. Notably, the trend is opposite to that observed for InAs (with similar material properties as $\mathrm{InN}$ ) NWs $[35,36]$, and also InN thin films $[19,20]$, where mobility has been found to decrease for increasing electron concentration.

In conclusion, InN NWs grown by CVD have been observed to exhibit interesting structural and electrical properties. The NWs, which were mostly coplanar, were found to bend spontaneously, or upon finding an obstacle in their growth path, at angles that are close multiples of $30^{\circ}$. Microfabricated $\mathrm{SiO}_{2}$ barriers forced multiple-NW deflections, resulting in $\mathrm{V}$-and $\mathrm{W}$-shaped structures. The electrical properties of the NWs were found to be excellent, with the NW FETs exhibiting drain current saturation, observed for the first time. The mobility, conductivity and carrier concentration were all found to increase with decreasing NW diameter, which can be modeled by considering NW core and surface conduction paths. Structural control of InN NWs, coupled with their superior electrical properties, can open up new applications for them in nanoelectronics and NEMS-based sensor arrays, probes and switches.

\section{Acknowledgments}

This work was performed with generous support from the National Science Foundation (grant no. ECCS-0846898) and Army Research Office (grant no. W911NF-08-0299). We would also like to acknowledge Drs Ghoshroy and Douglas Blom of the USC Electron Microscopy Center for their help with TEM measurements, and Dr Samir Garzon for helpful discussions.

\section{References}

[1] Ambacher O et al 1999 J. Appl. Phys. 85 3222-33

[2] Polyakov V M and Schwierz F 2006 Appl. Phys. Lett. 88032101

[3] Hullavarad S S et al 2006 J. Electron. Mater. 35 777-94

[4] Matsuoka T, Okamoto H, Nakao M, Harima H and Kurimoto E 2002 Appl. Phys. Lett. 81 1246-8

[5] Wu J, Walukiewicz W, Yu K M, Ager J W III, Haller E E, Lu H, Schaff W J, Saito Y and Nanishi Y 2002 Appl. Phys. Lett. 80 3967-9

[6] Mahboob I, Veal T D, Piper L F J, McConville C F, Lu H, Schaff W J, Furthmuller J and Bechstedt F 2004 Phys. Rev. B 69201307

[7] Mahboob I, Veal T D, McConville C F, Lu H and Schaff W J 2004 Phys. Rev. Lett. 92036804

[8] Colakerol L et al 2006 Phys. Rev. Lett. 97237601

[9] Zhang J, Zhang L, Peng X and Wang X 2002 J. Mater. Chem. 12802

[10] Johnson M C, Lee C J, Bourret-Courchesne E D, Konsek S L, Aloni S, Han W Q and Zettl A 2004 Appl. Phys. Lett. 855670

[11] Chen J, Cheng G, Stern E, Reed M A and Avouris P 2007 Nano Lett. 7 2276-80

[12] Chang C Y, Chi G C, Wang W M, Chen L C, Chen K H, Ren F and Pearton S J 2005 Transport properties of InN nanowires Appl. Phys. Lett. 87093112

[13] Cheng G, Stern E, Turner-Evans D and Reed M A 2005 Appl. Phys. Lett. 87253103

[14] Wilke I, Ascazubi R, Lu H and Schaff W J 2009 Appl. Phys. Lett. 93221113

[15] Tang T, Han S, Jin W, Liu X, Li C, Zhang D, Zhou C, Chen B, Han J and Meyyapan M 2004 J. Mater. Res. $19423-6$ 
[16] Liang C H, Chen L C, Hwang J S, Chen K H, Hung Y T and Chen Y F 2002 Appl. Phys. Lett. 81 22-4

[17] Vaddiraju S, Mohite A, Chin A, Meyyappan M, Sumanasekera G, Alphenaar B W and Sunkara M K 2005 Nano Lett. 5 1625-31

[18] Yin L W, Bando Y, Golberg D and Li M S 2004 Adv. Mater. 161833

[19] Jones R E, Li S X, Haller E E, van Genuchten H C M, Yu K M, Ager J W III, Liliental-Weber Z, Walukiewicz W, Lu H and Schaff W J 2007 Appl. Phys. Lett. 90162103

[20] Thakur J S, Naik R, Naik V M, Haddad D, Auner G W, Lu H and Schaff W J 2006 J. Appl. Phys. 99023504

[21] Huang Y, Duan X, Cui Y and Lieber C M 2002 Nano Lett. 2 101-4

[22] Cai Z, Garzon S, Chandrashekhar M V S, Webb R A and Koley G 2008 J. Electron. Mater. 37 585-92

[23] Chang C Y, Chi G C, Wang W M, Chen L C, Chen K H, Ren F and Pearton S J 2006 J. Electron. Mater. 35 738-43

[24] Chaudhry A and Islam M S 2008 J. Nanosci. Nanotechnol. $8222-7$

[25] Richter T, Lüth H, Schapers T, Meijers R, Jeganathan K, Hernandez S E, Calarco R and Marso M 2009 Nanotechnology 20405206
[26] Werner F, Limbach F, Carsten M, Denker C, Malindretos J and Rizzi A 2009 Nano Lett. 9 1567-71

[27] Cha H, Wu H, Chandrashekhar M V S, Choi Y C, Chae S, Koley G and Spencer M G 2006 Nanotechnology 17 1264-71

[28] Liu J, Cai Z and Koley G 2009 J. Appl. Phys. 106124907

[29] Dayeh S A, Susac D, Kavanagh K L, Yu E T and Wang D 2008 Nano Lett. $83114-9$

[30] Khanal D R and Wu J 2007 Nano Lett. 7 2778-83

[31] Petersen G, Hernandez S E, Calarco R, Demarina N and Schaepers Th 2009 Phys. Rev. B 80125321

[32] Aravind K, Su Y W, Ho I L, Wu C S, Chang-Liao K S, Su W F, Chen K H, Chen L C and Chen C D 2009 Appl. Phys. Lett. 95092110

[33] Hamaguchi C 2001 Basic Semiconductor Physics (Berlin: Springer) pp 375-77

[34] Kane B E, Pfeiffer L N, West K N and Harnett C K 1993 Appl. Phys. Lett. 63 2132-4

[35] Scheffler M, Nadj-Perge S, Kouwenhoven L P, Borgstrom M T and Bakkers E P A M 2009 J. Appl. Phys. 106124303

[36] Ford A C, Ho J C, Chueh Y, Tseng Y, Fan Z, Guo J, Bokor J and Javey A 2009 Nano Lett. $9360-5$ 